\title{
Payload dropping control of an unmanned quadrotor helicopter based on backstepping controller
}

\author{
Jing Qiao*, Zhixiang Liu, and Youmin Zhang \\ Concordia University, Montreal, H3G 1M8, Quebec, Canada
}

\begin{abstract}
Quadrotors have generated considerable interest in both the control community due to their simple dynamics and widely applications because of their advantages over regular air vehicles. Unlike other control algorithms that tend to linearize nonlinear systems, backstepping control does not work to cancel the nonlinearities in the system. This leads to more flexible designs since some of the nonlinear terms can contribute to the stability of the system. The backstepping control is implemented in this paper for the robustness of the quadrotor helicopter in the payload dropping process. This control algorithm is implemented on the parameters of an unmanned quadrotor helicopter platform known as Qball-X4 available at the Networked Autonomous Vehicles Lab of Concordia University. Simulation results carried out using a nonlinear model, and wherein the performance achieved with this control strategy is shown.
\end{abstract}

\section{Introduction}

Unmanned helicopters have become increasingly popular for various purposes concerning future commercial and military applications [1,2], either integrated with current manned aircraft or playing the role individually. The investigated and potential application areas include aerial surveillance [3,4], border patrol [5], environmental monitoring, search and exploration for unsecured regions [6], and search and rescue in the events of accidents and natural disasters in flood, earthquake, nuclear and radiation accidents $[7,8]$. Furthermore, monitoring and preventing forest fires and high building fires in urban areas $[9,10]$, surveillance of pipelines leakage [11], and other circumstances under which are highly risky for human pilots have also raised the need for the employment of unmanned systems for such applications.

Trajectory tracking with payload dropping is typical flight maneuver in using unmanned helicopters. Successful fulfillment of this mission is closely tied to the intervention of autopilot or autonomous control systems. This fact has motivated the research and development of new control algorithms for such applications with UAVs. Initially started with simple control algorithms such as PID control [12,13] or LQR control [14], however, simple linear methods were proved not to be promising for the nonlinear quadrotor system. The problem of nonlinear control design has been addressed using several methods such as

\footnotetext{
* Corresponding author: josephchiao250@gmail.com
} 
feedback linearization [15], sliding mode control [16], model predictive control (MPC) [17], and back-stepping control [18].

Nevertheless, among most of the studies concerning these control approaches, maximum take-off weight has always been assumed to be constant during the entire flight and the effects of either gradual or abrupt mass variation over the period of flight have not been widely investigated in the existing literature. The issue of mass variation caused by payload dropping is of much concern since some specific applications like search and rescue or fire-fighting, abrupt or gradual mass variation is inevitable. The study in this aspect for the benefits of such a practical application of the quadrotor was the main motivations for this work. In view of popularity and advantages of backstepping control strategy, in this paper, a backstepping controller is designed and implemented on a quadrotor helicopter UAV.

\section{System modeling of quadrotor}

\subsection{Qball-X4 quadrotor UAV}

The quadrotor UAV which is as the object of this study is the Qball-X4 [13] as shown in Fig. 1 which is at the Network Autonomous Vehicle (NAV) Lab in the Department of Mechanical and Industrial Engineering of Concordia University and was developed by Quanser Inc. partially under the financial support of NSERC (Natural Sciences and Engineering Research Council of Canada) in association with an NSERC Strategic Project Grant led by Concordia University since 2007. The quadrotor UAV is enclosed within a protective carbon fiber ball-shape cage (therefore a name of Qball-X4) to ensure safe operation. It uses four $10 \times 4.7$ inch propellers and standard RC motors and speed controllers.

The motors of the Qball-X4 are out-runner brushless motors. The generated thrust $T_{i}$ of the No.i motor is related to the No.i PWM input $u_{i}$ by a first-order linear transfer function as follows:

$$
\mathrm{T}_{i}=k \frac{\omega}{s+\omega} u_{i}
$$

where $i=1,2,3,4$ and $k$ is a positive gain and $\omega$ is the motor bandwidth. $k$ and $\omega$ are theoretically the same for the four motors but this may not be the case in practice. It should be noted that $u_{i}=0$ corresponds to zero thrust and $u_{i}=0.05 \mathrm{~ms}$ corresponds to the maximal thrust that can be generated by the No.i motor.

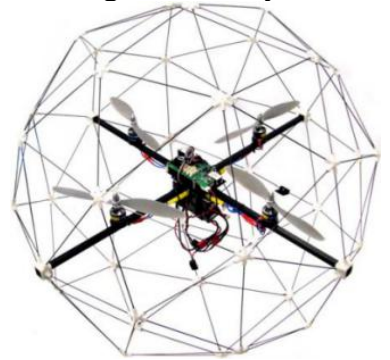

Fig. 1. The Qball-X4 quadrotor UAV.

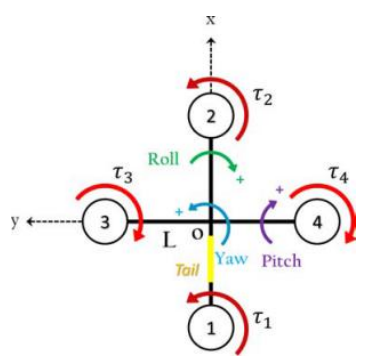

Fig. 2. Schematic representation of the Qball-X4

\subsection{System modeling of quadrotor helicopter}

A quadrotor is an under actuated aircraft with fixed pitch angle as shown in Figure 2. Having Quadrotors with fixed angles makes quadrotor has four input forces which are 
basically the thrust provided by each propellers. The motors and propellers are configured in such a way that the back and front (1 and 2) motors spin clockwise (thus inducing two counterclockwise torques on the body) and the left and right (3 and 4) spin counterclockwise (thus inducing two clockwise torques on the body). Each motor is located at a distance $\mathrm{L}$ from the center of mass o $(0.2 \mathrm{~m})$ and when spinning, a motor produces a torque $\tau_{\mathrm{i}}$ which is in the opposite direction of motion of the motor as shown in Fig. 2. Forward (backward) motion is maintained by increasing (decreasing) speed of front (rear) rotor speed while decreasing (increasing) rear (front) rotor speed simultaneously which means changing the pitch angle. Left and right motion is accomplished by changing roll angle by the same way. The front and rear motors rotate counter-clockwise while other motors rotate clockwise so yaw command is derived by increasing (decreasing) counterclockwise motors speed while decreasing (increasing) clockwise motor speeds.

Suppose the lift force generated by the $i$-th propeller in the quadrotors is $F_{i}(i=1,2,3,4)$, and $\tau_{1}, \tau_{2}, \tau_{3}, \tau_{4}$ are torque generated by each motor. The force acting on the UAV in the airframe coordinate system is:

where

$$
F_{B}=\left[0,0, U_{1}\right]^{T}
$$

$$
U_{1}=F_{1}+F_{2}+F_{3}+F_{4}
$$

Based on Newton's second law, the rectilinear motion model and angular motion model of the quadrotors were obtained as,

$$
\begin{gathered}
{\left[\begin{array}{c}
\ddot{X} \\
\ddot{Y} \\
\ddot{Z}
\end{array}\right]=\frac{1}{m}\left[\begin{array}{c}
U_{1}(\sin \phi \sin \psi+\cos \phi \cos \psi \sin \theta)-K_{1} \dot{X} \\
U_{1}(\cos \phi \sin \theta \sin \psi-\cos \psi \sin \phi)-K_{1} \dot{Y} \\
U_{1}(\cos \phi \cos \theta)-K_{2} \dot{Z}-m g
\end{array}\right]} \\
\left\{\begin{array}{c}
\ddot{\varphi}=\left[L\left(F_{4}-F_{2}\right)+\dot{\theta} \dot{\psi}\left(I_{y}-I_{z}\right)\right] / I_{y} \\
\ddot{\theta}=\left[L\left(F_{3}-F_{1}\right)+\dot{\varphi} \dot{\psi}\left(I_{z}-I_{\mathrm{x}}\right)\right] / I_{y} \\
\ddot{\psi}=\left[\left(\tau_{2}+\tau_{4}-\tau_{1}-\tau_{3}\right)+\dot{\varphi} \dot{\theta}\left(I_{x}-I_{y}\right)\right] / I_{z}
\end{array}\right.
\end{gathered}
$$

The quadrotor is a 4 input, 6 output system and the coupling of 4 inputs to 6 outputs is too strong, so, we set intermediate inputs as:

$$
\left\{\begin{array}{c}
U_{1}=F_{1}+F_{2}+F_{3}+F_{4} \\
U_{2}=F_{4}-F_{2} \\
U_{3}=F_{3}-F_{1} \\
U_{4}=\tau_{2}+\tau_{4}-\tau_{1}-\tau_{3}
\end{array}\right.
$$

Using above formulas, the nonlinear dynamic model of the quadrotor can be obtained as,

$$
\begin{aligned}
& \ddot{X}=\left[U_{1}(\cos \varphi \sin \theta \cos \psi+\sin \varphi \sin \psi)-K_{1} \dot{X}\right] / m \\
& \ddot{Y}=\left[U_{1}(\sin \psi \sin \theta \cos \varphi-\cos \psi \sin \varphi)-K_{2} \dot{Y}\right] / m \\
& \ddot{Z}=\left[U_{1}(\cos \varphi \cos \theta)-m g-K_{3} \dot{Z}\right] / m \\
& \ddot{\varphi}=\left[U_{2} L+\dot{\theta} \dot{\psi}\left(I_{y}-I_{z}\right)\right] / I_{x} \\
& \ddot{\theta}=\left[U_{3} L+\dot{\varphi} \dot{\psi}\left(I_{z}-I_{x}\right)\right] / I_{y} \\
& \ddot{\psi}=\left[U_{4}+\dot{\varphi} \dot{\theta}\left(I_{y}-I_{z}\right)\right] / I_{z}
\end{aligned}
$$

The dynamic equation (7) shows that the quadrotor UAV flight dynamics model has the characteristics of underactuated, nonlinear, uncertainty and strong coupling. 


\section{Backstepping controller design}

Unlike other control algorithms that tend to linearize nonlinear, backstepping does not work to cancel the nonlinearities in the system. This leads to more flexible designs since some of the nonlinear terms can contribute to the stability of the system. The backstepping control requires that the dynamic model of the system satisfies the strict feedback form, that is, the form of the inverted triangle. The dynamic model of the horizontal position subsystem of the quadrotor unmanned aerial vehicle shows that the control input of the system directly affects the roll angle and pitch angle of the aircraft, and the position and line velocity signals are not included in the dynamic model of the attitude angle. The dynamic model of the aircraft's horizontal position is directly related to the attitude angle of the aircraft, so it can be shown that the horizontal position subsystem of the quadrotor unmanned aerial vehicle satisfies the strict feedback form.

\subsection{Backstepping controller design for quadrotor UAV}

Due to the strict symmetry of the quadrotor helicopter structure, the Quadrotor height control channel and yaw control channel are relatively independent, and its horizontal control is controlled by the underdrive of rolling angle and pitch angle. When virtual input vector $U=\left(U_{2}, U_{3}, U_{4}\right)$ is known, three attitude angles and their angular velocities can be obtained. If the $U_{1}$ is also known, then the position and speed of the aircraft will be obtained. Obviously, the attitude loop and the position loop are semi-coupled. The position loop is the inner loop and the position is the outer loop.

Take the state variables as $x=(\phi, \dot{\phi}, \theta, \dot{\theta}, \psi, \dot{\psi}, z, \dot{z}, x, \dot{x}, y, \dot{y})$, that

$$
\begin{aligned}
& \text { is } x_{1}=\phi, x_{2}=\dot{x}_{1}=\dot{\phi} ; x_{3}=\theta, x_{4}=\dot{x}_{3}=\dot{\theta} ; x_{5}=\psi, x_{6}=\dot{x}_{5}=\dot{\psi} \text {; } \\
& x_{7}=z, x_{8}=\dot{x}_{7}=\dot{z} ; x_{9}=x, x_{10}=\dot{x}_{9}=\dot{x} ; x_{11}=y, x_{12}=\dot{x}_{11}=\dot{y}
\end{aligned}
$$

The mathematical model of the Quadrotor is written as a strict feedback format,

$$
f(x, U)=\left\{\begin{array}{l}
x_{2}=\dot{x}_{1} \\
\dot{x}_{2}=a_{1} \cdot x_{4} \cdot x_{6}+b_{1} \cdot U_{2} \\
\dot{x}_{3}=x_{4} \\
\dot{x}_{4}=a_{2} \cdot x_{2} \cdot x_{6}+b_{2} \cdot U_{3} \\
\dot{x}_{5}=x_{6} \\
\dot{x}_{6}=a_{3} \cdot x_{2} \cdot x_{4}+b_{3} \cdot U_{4} \\
\dot{x}_{7}=x_{8} \\
\dot{x}_{8}=\cos x_{1} \cdot \cos x_{3} \cdot U_{1} / m-g \\
\dot{x}_{9}=x_{10} \\
\dot{x}_{10}=U_{x} \cdot U_{1} / m \\
\dot{x}_{11}=x_{12} \\
\dot{x}_{12}=U_{y} \cdot U_{1} / m
\end{array}\right.
$$

where $a_{1}=\left(I_{y}-I_{z}\right) / I_{x}, b_{1}=L / I_{x} ; a_{2}=\left(I_{z}-I_{x}\right) / I_{y}, b_{2}=L / I_{y}$;

$a_{3}=\left(I_{x}-I_{y}\right) / I_{z}, b_{3}=L / I_{z} ; U_{x}=(\cos \phi \sin \theta \cos \psi+\sin \phi \sin \psi) ;$

$U_{y}=(\cos \phi \sin \theta \sin \psi-\sin \phi \cos \psi)$.

In order to reduce the difficulty of the design of the system controller, the whole system is divided into 6 relatively independent control channels. Where, position loop contains a height controlled channel, horizontal $x, y$ control channel. And the attitude loop contains the roll control channel, the pitch control channel and the yaw control channel. The backstepping method is applied in each relatively independent channel. 


\subsection{System parameters}

The MATLAB/Simulink simulation tool is used to simulate the control algorithm for quadrotor helicopter. Some of the basic parameters for QballX4 quadrotor UAV model simulation are shown in Table 1.

Table 1. Basic parameters of the quadrotor UAV Qball-X4.

\begin{tabular}{|c|c|}
\hline Parameter & value \\
\hline$K$ & $120 \mathrm{~N}$ \\
\hline$\omega$ & $15(\mathrm{rad} / \mathrm{sec})$ \\
\hline$I x$ & $0.03\left(\mathrm{~kg} \cdot \mathrm{m}^{2}\right)$ \\
\hline$I y$ & $0.03\left(\mathrm{~kg} \cdot \mathrm{m}^{2}\right)$ \\
\hline$I z$ & $0.04\left(\mathrm{~kg} \cdot \mathrm{m}^{2}\right)$ \\
\hline$m$ & $1.4(\mathrm{Kg})$ \\
\hline$K$ & $4(\mathrm{~N} \cdot \mathrm{m})$ \\
\hline$l$ & $0.2(\mathrm{~m})$ \\
\hline$g$ & $9.8(\mathrm{~N} / \mathrm{kg})$ \\
\hline
\end{tabular}

Normally, if the desired input signal is small, select the larger R matrix, which forces the input signal to be smaller. Otherwise, the objective function value will increase and will not achieve the optimization requirements. Compared with the results of simulation tests, a larger $c_{i}$ is likely to lead to a smaller overshoot. At the same time, the overshoot of position will also increase. Considering the safety of actual flight, take

$$
\begin{aligned}
& c_{i}=1.5,(i=1,2,3,4,5,6) \\
& c_{j}=1.25,(j=7,8,9,10,11,12)
\end{aligned}
$$

\section{Simulation results}

In this paper, the system simulation based on the Matlab/Simulink is carried out to verify the design result of backstepping controller. In the simulation experiment, the initial state of the Qball-X4 is set as, altitude $\mathrm{z}$ is $0 \mathrm{~m}$, roll angle $\phi$, pitch angle $\theta$, and yaw angle $\psi$ are all 0 degree. And while the Qball-X4 finishes climbing and is in a rotating trajectory, the desired altitude $\mathrm{z}_{\mathrm{d}}$ is $1 \mathrm{~m}$ and the rotating diameter is $1 \mathrm{~m}$. The payload of $300 \mathrm{~g}$ in the Qball$\mathrm{X} 4$ is dropping at the 20 second. Simulation time is set to 40 seconds. The command for the backstepping controller of the Qball-X4 helicopter is shown in figure 3. The focus of this study is mainly on the flight stability when the payload is dropped from the Qball-X4.

Figure 4 to figure 7 show the flying path, flying attitude and the total thrust of Qball-X4 when payload is dropping with the backstepping controller. It could be seen from simulation results that the backstepping controller was capable of keeping the desired height and trajectory.

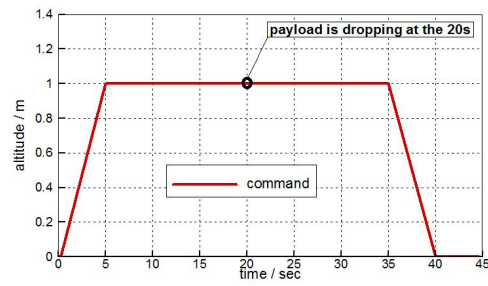

Fig. 3. Command for the Qball-X4 controller.

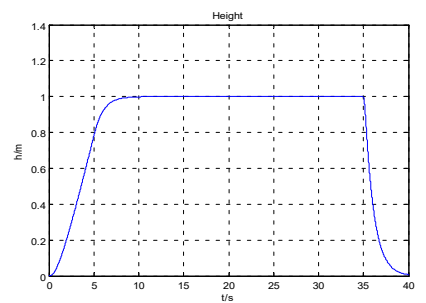

Fig. 4. The flying path of Qball-X4 for payload dropping at the $20 \mathrm{~s}$ with backstepping controller. 
The flying height in figure 4 indicates that the Qball-X4 eventually reach the desired altitude at the10s. It could also been found from the flying attitude of Qball-X4 that, at the moment when Qball-X4 arriving at desired height and the moment of Qball-X4 beginning landing, the roll angle and pitch angle of Qball-X4 are fluctuating. However these flying attitude fluctuating is restricted to a small extent, the fluctuating of the roll angle is smaller than 0.6 degrees, and the fluctuating of the pitch angle is in the range \pm 2 degrees. And the roll angle also fluctuates at the moment of payload drop.

It could also be obviously seen from figure 8 that total thrust abrupt change of Qball-X4 when payload drops. Figure 8 is a three dimensional trajectory map of a Quadrotor helicopter trajectory tracking. As can be seen from this figure, the aircraft starts at points $(0$, $0,0)$, then climbs up to the plane of $z=1$, and this plane travels along a circle of 1 meters in diameter. And, after the payload drop, the quadrotor helicopter gradually descends to startspoints $(0,0,0)$. Therefore, the control design makes the Quadrotor UAV achieve the trajectory tracking effect.

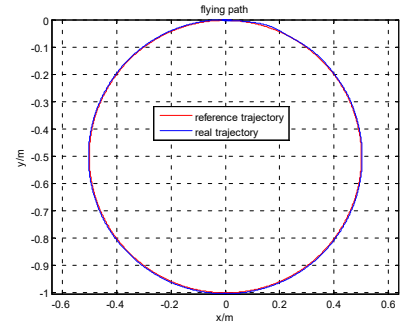

Fig. 5. The $x-y$ position change of Qball-X4 for payload dropping at the 20 s with backstepping controller.

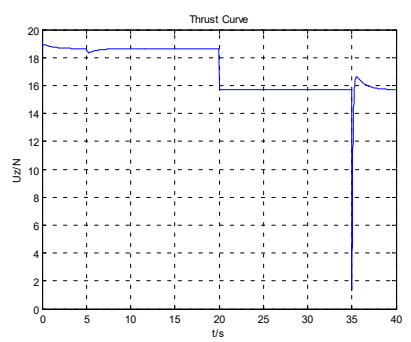

Fig. 7. The total thrust of Qball-X4 for payload dropping at the $20 \mathrm{~s}$ with backstepping controller.

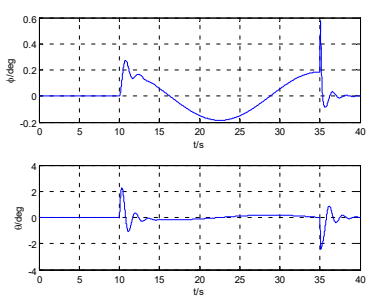

Fig. 6. The flying attitude of Qball-X4 for payload dropping at the 20 s with backstepping controller.

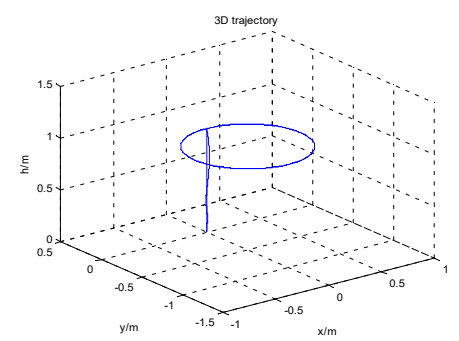

Fig. 8. 3D trajectory of Qball-X4.

\section{Conclusion}

In this paper, a robust backstepping control strategy has been presented for the quadrotor to obtain trajectory tracking and payload dropping by controlling its attitude and position simultaneously. The flight simulation experiments of the quadrotor UAV was carried out in the paper. The simulation results verify the effectiveness of the proposed backstepping controller. It is found that the backstepping controller is able to control the quadrotor in the presence of payload dropping and to make the quadrotor UAV achieve the trajectory tracking effect. With the backstepping control, the position $\{\mathrm{x} \mathrm{y}, \mathrm{z}\}$ and attitude tracking was successfully achieved in the climbing, hovering, payload dropping and descending of 
a quadrotor helicopter.

It should be indicated that it is difficult to model the quadrotor UAV accurately when there is uncertainty in the model such as the abrupt mass variation over the period of flight for payload dropping. The control design of this paper only investigated the problem of aircraft control when there is a payload dropping in certain smooth flight trajectory using backstepping controller. The simulation results indicate that the roll angle will fluctuates at the moment of payload drop.

It is well known that the system should maintain acceptable performance with partial actuator faults using fault tolerant control strategies for the control of UAV. Therefore, in the future, with adopting the backstepping control approach and utilizing the quadrotor mathematical model, a fault tolerant control law could be further designed and evaluated by single, triple or even quadruple partial actuator faults.

\section{References}

1. Y. M. Zhang, A. Chamseddine, C. A. Rabbath, B. W. Gordon, C.-Y. Su, S. Rakheja, C. Fulford, J. Apkarian and P. Gosselin, Development of advanced FDD and FTC techniques with application to an unmanned Quadrotor helicopter testbed, J. Franklin Inst. 350(9) (2013) 2396- 2422.

2. F. Lin, K. Z. Y. Ang, F. Wang, B. M. Chen, T. H. Lee, B. Yang, M. Dong, X. Dong, J. Cui, S. K. Phang, B. Wang, D. Luo, K. Peng, G. Cai, S. Zhao, M. Yin and K. Li, Development of an unmanned coaxial rotorcraft for the DARPA UAVForge challenge, Unmanned Syst. 1(2) (2013) 211-245.

3. J. Keller, D. Thakur, V. Dobrokhodov, K. Jones, M. Pivtoraiko, J. Gallier, I. Kaminer and V. Kumar, A computationally efficient approach to trajectory management for coordinated aerial surveillance, Unmanned Syst. 1(1) (2013) 59-74.

4. P. C. Niedfeldt, B. T. Carroll, J. A. Howard, R. W. Beard, B. S. Morse and S. Pledgie, Enhanced UAS surveillance using a video utility metric, Unmanned Syst. 1(2) (2013) 277-296.

5. C. C. Haddal and J. Gertler, Homeland security: Unmanned aerial vehicles and border surveillance, CRS Report for Congress, Congressional Research Service (2010).

6. J. Hu, J. Xu and L. Xie, Cooperative search and exploration in robotic networks, Unmanned Syst. 1(1) (2013) 121-142.

7. M. K. Habib and Y. Baudoin, Robot-assisted risky intervention, search, rescue and environmental surveillance, Int. J. Adv. Robot. Syst. 7(1) (2010) 1-8.

8. T. Skrzypietz, Unmanned aircraft systems for civilian missions, BIGS Policy Paper No. 1 (2012).

9. R. W. Beard, T. W. McLain, D. B. Nelson, D. Kingston and D. Johanson, Decentralized cooperative aerial surveillance using fixed-wing miniature UAVs, Proc. IEEE 94(7) (2006) 1306-1324.

10. D. W. Casbeer, D. B. Kingston, R. W. Beard and T. W. McLain, Cooperative forest fire surveillance using a team of small unmanned air vehicles, Int. J. Syst. Sci. 37(6) (2006) 351-360.

11. H. Dieter, Z. Werner, S. Gunter and S. Peter, Monitoring of gas pipelines: A civil UAV application, Aircraft Eng. Aerosp. Technol. 77(5) (2005) 352-360.

12. B. Erginer and E. Altug, Modeling and PD control of a Quadrotor VTOL vehicle, IEEE Intelligent Vehicles Symp. (2007), pp. 894-899.

13. A. Wahyudie, T. B. Susilo and H. Noura, Robust PID controller for quadrotors, J. 
Unmanned Syst. Technol. 1(1) (2013) 14-19.

14. K. Oner, E. Cetinsoy, M. Unel, M. Aksit, I. Kandemir and K. Gulez, Dynamic model and control of a new Quadrotor UAV with tilt-wing mechanism, World Academy of Science, Engineering and Technology (2008).

15. M. O. Efe, Robust low altitude behavior control of a Quadrotor rotorcraft through sliding modes, Mediterranean Conf. on Control and Automation (2007).

16. H. Bouadi, M. Bouchoucha and M. Tadjine, Sliding mode control based on backstepping approach for an UAV type-Quadrotor, World Academy of Science, Engineering and Technology (2007).

17. M. Abdolhosseini, Y. M. Zhang and C. A. Rabbath, An efficient model predictive control scheme for an unmanned Quadrotor helicopter, J. Int. Robot. Syst. 70(1) (2013) 27-38.

18. Madani, T. and Benallegue, A. (2006) Backstepping Control for a Quadrotor Helicopter. Proceedings of the 2006 IEEE/RSJ International Conference on Intelligent Robots and Systems, Beijing, 9-15 October 2006, 3255-3260. 